\title{
En terreno vedado: género, traducción y censura. El caso de Brokeback Mountain
}

\author{
Cristina Gómez Castro y María Pérez L. Heredia \\ Universidad de León / Universidad del País Vasco \\ cristina.gomez@unileon.es; maria.perezl@ehu.es
}

\begin{abstract}
Resumen: A la hora de traducir no solo hay que tener en cuenta la diferencia lingüística, sino especialmente la cultural y, cómo no, también la ideológica. En este sentido, nuestra aportación pretende analizar las implicaciones de la traducción a castellano del relato Brokeback Mountain (Proulx, 1997) y la reescritura intersemiótica que da lugar a la película homónima (Lee, 2005), centrándose especialmente en la manipulación y trasvase de los estereotipos de género. Para ello, se atenderá a las cuestiones ideológicas, de censura y culturales, en un estudio de caso que se presenta especialmente interesante en tanto que nos permite reactualizar la actividad traductológica en relación con la cual no caben visiones ingenuas de mera transferencia.
\end{abstract}

Palabras clave: género; estereotipos; ideología; censura.

\begin{abstract}
When translating, not only linguistic difference should be taken into consideration, but also and in particular, the cultural and ideological one. Our article sets out to analyze the implications of the translation into Spanish of the short story Brokeback Mountain (Proulx, 1997) and also of the inter-semiotic rewriting which turned it into the film with the same name (Lee, 2005). In order to do that, it will focus on the manipulation and transference of gender stereotypes. Ideological, censoring and cultural issues will be at stake in a case study that is specially interesting in that it allows us to revisit the translating activity as one in which no naive visions of mere transference have a say anymore.
\end{abstract}

Keywords: gender; stereotypes; ideology; censorship. 



\section{Introducción}

A finales del año 2005, el estreno de una película sacudía las pantallas, y las entrañas, del mundo entero: definitivamente, Brokeback Mountain no era un western más. La historia de amor homosexual entre dos vaqueros agitaba las conciencias al tiempo que parecía dar jaque a un buen número de estereotipos que, durante décadas, había marcado el devenir de Hollywood y, en consecuencia, del planeta americano que habitamos (apud Verdú, 1996). ¿Acaso estábamos presenciando el fin del hombre Marlboro? El icono de la masculinidad por excelencia, cuanto menos, se tambaleaba, y los espectadores de medio mundo eran testigos de ello, por obra y gracia de la traducción. Parecía imposible que la homofobia predominante en la industria cinematográfica hubiera permitido semejante atrevimiento ideológico, pero lo cierto es que la cinta, con explícitas escenas de sexo entre dos tipos duros, llegaría a recibir el aplauso (casi) unánime de público y crítica ${ }^{1}$. Lo que entonces fue provocación, subversión y agitación, es hoy normalidad; sin duda alguna, Brokeback Mountain allanó el camino al nuevo cine, a la nueva televisión, que incorpora en la actualidad personajes y temas gay, de todo género y condición, con naturalidad.

Por la parte que nos toca, es inevitable que los Estudios de Traducción presten atención a los elementos ideológicos subyacentes a cualquier texto y, de esta forma, revisitar y reconceptualizar antiguas visiones que consideraban el hecho traductor como un mero puente entre culturas, una pasarela que, al recorrerse, debía convertirse en un producto lo más neutro e invisible posible. Es nuestro propósito detenernos en el hecho de que, al traducir, no solo hay que tener en cuenta la diferencia lingüística, sino especialmente la cultural y, cómo no, también la ideológica. En este sentido, nuestra aportación pretende analizar las implicaciones de las traducciones del relato Brokeback Mountain (Proulx, 1997) y la reescritura intersemiótica que lo convertiría en la película homónima (Lee, 2005), centrándonos especialmente en

\footnotetext{
${ }^{1}$ A pesar del éxito, un Hollywood ciertamente hipócrita no concedió todos los honores a la cinta al negarle el Óscar a la mejor película: en 18 de los anteriores 22 años de los premios de la academia, dicho Óscar se había concedido a la película ganadora del Globo de Oro a Mejor Película, incumpliéndose dicha premisa en el caso de Brokeback Mountain, ya que la película ganadora fue Crash, ni siquiera nominada para dicho Globo de Oro (Karger, 2006).
} 
la manipulación y reescritura de los estereotipos en el terreno de la traducción literaria y audiovisual. Atenderemos a las cuestiones ideológicas, de censura y culturales pero sin olvidar, por otro lado, que tanto la traducción literaria como la audiovisual se relacionan de manera muy frecuente entre ellas y también, obligatoriamente, con la reescritura intersemiótica, otra forma de auténtica traducción que no puede obviarse en un estudio de estas características. Este artículo busca completar el estudio traductológico completo de Brokeback Mountain ${ }^{2}$ y resulta un caso especialmente interesante y paradigmático, en tanto que nos permite reactualizar la actividad traductológica en relación con la cual no caben visiones ingenuas de mera transferencia, una actividad que es acción y que, en palabras de Gentzler and Tymozcko (2002: xxi): “[...] is not simply an act of faithful reproduction but, rather, a deliberate and conscious act of selection, assemblage, structuration, and fabrication - and even, in some cases, of falsification, refusal of information, counterfeiting, and the creation of secret codes", tal y como veremos en este estudio.

Pasamos pues a continuación a ver, en primer lugar, el relato y su traducción al castellano, para después ofrecer un breve análisis del trasvase intersemiótico del mismo al cine.

\section{El relato}

Annie Proulx publicó por primera vez Brokeback Mountain el 13 de octubre de 1997 en el semanario The New Yorker ${ }^{3}$ como un relato aislado, ya que no sería hasta dos años después que este pasaría a formar parte de su colección de historias titulada Close Range: Wyoming Stories, un conjunto de relatos ubicados en el Estado norteamericano de Wyoming y que recrean varias vidas rústicas y violentas. Proulx había tardado en escribir la historia unas seis semanas y, según la misma autora, la observación durante muchos años de la Norteamérica rural y profunda había sido su principal fuente de inspiración para escribir sobre la controvertida temática de la homosexualidad en un mundo de vaqueros.

\footnotetext{
${ }^{2}$ El estudio de la traducción audiovisual de la película se ha llevado a cabo en "Más allá de las palabras: traducción audiovisual y estereotipos de género en Brokeback Mountain" (Pérez L.Heredia, M. \& Gómez Castro, C. En prensa).

${ }^{3}$ El relato tal y como se publicó la primera vez puede encontrarse en: http://www. newyorker.com/magazine/1997/10/13/brokeback-mountain.
} 
La acogida que el relato recibió no dejó a nadie indiferente, si bien las reacciones de mayor calado a la misma tuvieron lugar una vez que se hubo trasvasado a la gran pantalla en forma de película en el año $2005^{4}$. Esta sinergia entre literatura y cine suele ser común como fuente de ideas en el mundo audiovisual ${ }^{5}$ y en el caso de Brokeback Mountain elevó la fama del relato a la categoría de internacional y desencadenó su reedición y traducción masiva, pasando a ser el central de las Historias de Wyoming.

El argumento del relato es de sobra conocido: Ennis del Mar y Jack Twist, dos jóvenes que se conocen un verano mientras ejercen de cuidadores de ovejas al pie de una montaña, experimentan allí su primera relación homosexual que los deja marcados para siempre. Tras dicho tiempo, sus caminos se separan pero solo para volverse a juntar de forma intermitente desde entonces, continuando su idilio pero negándolo de cara a la sociedad, donde ejercen de heterosexuales, se casan y tienen hijos. Nunca vivirán como pareja: el miedo a una reacción homofóbica y su propia lucha interior se lo impide. El desenlace de la obra es desgarrador tanto para el lector como para el espectador y fuente del actual disgusto de la autora para con una obra y unos personajes que tanta vida cobraron para ella: no hace mucho declaró que deseaba no haber escrito la historia, debido al hastío provocado por la avalancha de fans que le han escrito con finales alternativos, sin aceptar el que ella le dio en su momento: "I wish I'd never written the story. It's just been the cause of hassle and problems and irritation since the film came out. Before the film it was all right" (Cox, 2009: en línea).

Alabada por unos y criticada por otros, lo que no puede sin duda negársele es su valor como relato agitador de la conciencia social, a pesar de las palabras de la autora cuando afirma que "I don't write to inspire social change, but I do like situations of massive economic or cultural change as a background. We think of change as benign, but it chews some people up and spits them out. And fiction can bring about

\footnotetext{
${ }^{4}$ Ang Lee fue el director y los encargados de convertir la obra en un guion cinematográfico Larry McMurtry y Diana Ossana, con la colaboración de la propia Proulx. La obra, tal y como se verá en más detalle en este artículo, obtuvo gran éxito y fue galardonada con tres Óscar y cuatro Globos de Oro.

${ }^{5}$ Es lo que se conoce de forma más general como tie-in, y muy frecuente en el caso de las películas basadas en libros o relatos.
} 
change" (ibid.). Ese cambio del que hablaba Proulx fue sin duda patente en la obra que nos ocupa, revulsivo que no dejaría a nadie indiferente ${ }^{6}$.

\subsection{Una cuestión de género}

Podría decirse que Proulx, mujer y de Nueva Inglaterra, se presenta como forastera por partida doble en un mundo fundamentalmente de hombres ubicado en el Oeste norteamericano como el que aparece reflejado en Brokeback Mountain. Cuando comenzó a escribir historias de ficción fueron los propios editores, en una actitud claramente sexista, los que le aconsejaron que cambiara su nombre a unas simples iniciales con el fin de que el público lector de las revistas donde se iban a publicar sus relatos, mayoritariamente masculino, no pensara que habían sido escritos por una mujer (Cox, 2009). Por dicha razón, algunas de sus obras aparecen firmadas como E.A. Proulx y otras como E. Annie hasta que finalmente ella misma decidió eliminar la E. y firmar como Annie Proulx. Estos inconvenientes, no obstante, no mermaron su capacidad productiva, que le llevó a ganar varios premios, entre ellos el codiciado PEN/Faulkner, convirtiéndose así en la primera mujer escritora en ganarlo, en este caso gracias a la novela Postcards, publicada en 1993.

El papel que las mujeres representan en sus historias cortas de Wyoming no es nunca central, ello debido fundamentalmente al hecho de que, tal y como ella misma admite, el trabajo más duro y el que más visibilidad tenía, fuese pescar o trabajar en el rancho, era llevado a cabo por hombres (Cox, 2009). No obstante, según Proulx reconocía (ibid.), se trataba más de una cuestión geográfica que de género, algo que parece funcionar del mismo modo en Brokeback Mountain, donde el centro de la historia son dos hombres. Un detalle que podría parecer perfectamente natural en una historia del Oeste como esta se convierte no obstante en un aspecto subversivo del género western y de todas sus convenciones. Así, tanto Ennis como Jack son a la vez "perpetuators and victims of the «cowboy myth»" (Campbell, 2009: 212). Si se ana-

\footnotetext{
${ }^{6}$ Ningún otro personaje gay ha sido tan influyente como Ennis y Jack de la adaptación cinematográfica, que inspiraron la empatía y entendimiento frente a la estereotipación despectiva que había sido la tónica hasta entonces (Roughton, 2014: 85). Tras ello, los derechos de los colectivos gays en Estados Unidos comenzaron a ser testigos de una mejoría que llevaría al propio presidente Barack Obama a anunciar su apoyo al matrimonio gay en 2012 (ibid.).
} 
lizan las trayectorias de ambos personajes en el relato, se observa cómo cada uno de ellos cumple con un papel que en principio parece a todas luces rompedor con el que deberían tener en un western al uso tradicional: para muchos, el western es "the American genre" (Spohrer, 2010: 31) y, por lo tanto, una subversión del mismo se ve como propia de la cultura norteamericana en general.

Dicha subversión comienza con el mismo rol de ambos como pastores de ovejas en lugar de vaqueros, a pesar de que en todo momento se los considera como lo último. La figura del centauro masculino que es pieza fundamental de una obra del Oeste queda de este modo rebajada a la categoría de pastor, con lo que la autora juega con dicha caracterización. Del mismo modo, los protagonistas de los westerns al uso son masculinos y se alejan del entorno de la ciudad, que los envuelve y los asfixia, censurando su historia de amor. En este caso, Jack y Ennis se alejan de la ciudad para adentrarse en la montaña, donde darán rienda suelta a sus instintos, algo para lo que no había lugar en los westerns clásicos. Lacónicos, serios y poco dados a formalismos, los vaqueros suelen ser sinceros y expresan abiertamente sus opiniones (González Sánchez, 2011: 10), algo que no se da en el caso de Jack ni, en especial, de Ennis, que no será hasta el final del relato cuando admita, y de forma entrecortada, sus verdaderos sentimientos. Inserta en una cultura profundamente homofóbica, la historia ve cómo sus personajes se niegan a aceptar lo que realmente les pasa, a pesar de que el arco de transformación de los arquetipos en un wéstern clásico nos indica que debería haber una evolución y una maduración paulatina, puesto que "los acontecimientos experimentados por los héroes poseerán la intensidad suficiente para alterar el equilibrio inicial del personaje" (ibíd.: 13). Dentro del esquema del género, Jack es más héroe que Ennis (Averbach, 2009: 149), ya que siempre está dispuesto a cumplir con su propio código de vida e intenta por todos los medios que se vayan a vivir juntos. Por el contrario, Ennis adoptará un papel más activo en las relaciones sexuales que ambos mantienen, donde Jack pasa a un segundo plano, cruzando de este modo Proulx las características de ambos (ibíd.).

Algunos la han considerado como una historia de amor en lugar de como un wéstern (Spohrer, 2010: 28), lo cual potencialmente atraería a una audiencia mayor. Ciertamente, el amor a lo "Romeo y Julieta" que se expresa en algunos de los momentos vividos por ambos personajes permite atisbar marcas de un verdadero relato de amor. Sea como sea, si 
se ha de enmarcar el relato, no ha de hacerse en un género determinado sino más bien en una época y un momento concretos, sin los que la obra no hubiera podido ser. Lejos de considerarla como atemporal, lo cual sería un error, la propia autora reconoce que la historia “isn't about Jack and Ennis. It's about homophobia; it's about a social situation; it's about a place and a particular mindset and morality" (Cox, 2009: en línea), un patriarcado y una sociedad homofóbica que forjarán la personalidad de los protagonistas y que ha de mantenerse para que la historia tenga el sentido que la autora quiso darle.

Así pues, a continuación pasaremos a ver cómo fue trasvasado el relato de inglés a español y publicado en la península, en traducción de María Corniero, para así poder dilucidar si las subversiones y giros que Proulx imprimió a su historia se ven reflejadas en la versión en español o por el contrario sufren algún tipo de alteración o cambio.

\subsection{El relato Brokeback Mountain en español: ¿(auto)censura?}

La primera traducción a español peninsular que encontramos de la obra corresponde a la realizada, tal y como se indica más arriba, por María Corniero en el año 2000 para la editorial Siglo XXI de España y como parte de En terreno vedado. Historias de Wyoming de Proulx. Se trata de una historia corta que, lingüísticamente, no sorprende ni por sus bellas frases ni por sus sutilezas, pero aun así engancha al lector desde el primer momento, al igual que lo hará con posterioridad la película. Tal y como expresaba la propia autora, en un relato, "every single word counts heavily" (Cox, 2009: en línea). Por esta razón, a la hora de analizar la traducción de la obra a nuestro idioma, se prestará sobre todo atención a aquellos aspectos que podían ser más susceptibles de trasvasar las ideas más subversivas del texto, tanto en cuestiones de género y su expresión y represión como en cuestiones de lenguaje, ya que es a través de este último como los personajes se caracterizan y por lo tanto se presentan al público lector.

En primer lugar nos centramos en la traducción de los aspectos de moral sexual y escenas de amor presentes en el relato, puesto que si hay algún aspecto que siempre ha sido objeto de censura este ha sido el sexo. No son muchas las ocasiones en las que Proulx nos explicita los encuentros de esta índole entre Jack y Ennis, pero sí lo suficientemente intensas como para dar idea de su pasión. 
Así, la escena del primer encuentro sexual entre ambos, que tiene lugar en la tienda durante una de sus frías noches en la montaña, nos presenta a un Ennis reacio al comienzo y activo después, siendo Jack el iniciador para pasar a ser pasivo una vez que Ennis toma las riendas. La traducción de Corniero mantiene la estructura del original y no se desvía ni intensificando ni atenuando el texto inglés, según la clasificación de Franco Aixelá y Abio Villarig (2009). La misma estrategia de literalidad será la que adopte en el resto de encuentros con tensión sexual entre ambos vaqueros, tanto el reencuentro en el que Alma es testigo inintencionado de su beso apasionado ("their mouths came together" (ibid.: 77)/“sus bocas se juntaron" (ibíd.: 15)) ${ }^{7}$ como en la escena que sigue a continuación con ellos dos en el motel ("within twenty minutes were in the Motel Siesta jouncing a bed" (ibid.: 78)/“en menos de veinte minutos estaban [sic] meneando una cama en el Motel Siesta" (ibid.: 16)). El último de sus encuentros que se reflejan en el relato corresponde a la reunión final entre ambos antes del fallecimiento de Jack: también aquí la estrategia traductora de Corniero es la de adecuarse a la expresión inglesa, intentando que la prosa de Proulx sea trasvasada a español de la forma más neutral posible.

Pero estos no son los únicos encuentros sexuales que aparecen en el relato, aunque sí los exclusivos de índole homosexual explícitamente indicados. El resto se referirá a Ennis y su todavía mujer, Alma, donde puede apreciarse la actitud mucho más indiferente y fría de este para con ella que para con Jack: "he rolled her over, did quickly what she hated" (ibid.: 77)/“"le dio media vuelta e hizo a toda prisa lo que ella detestaba (ibíd:: 13)". Poco a poco, la relación del matrimonio se deteriora y Alma le pide que use preservativos para no quedarse más embarazada, a lo cual Ennis se niega. Es entonces cuando Alma deja entrever sus primeros síntomas de rabia hacia él, a pesar de que lo hace solamente en sus pensamientos: "what you like to do don't make too many babies (ibid.: 79)"/"lo que a ti te gusta hacer no engendra muchos hijos (ibid.: 22)". Será más adelante cuando, una vez ya separados, le eche en cara sus encuentros con Jack, acusando a este de "nasty" (ibid.: 80), en español traducido como "marrano" (ibíd.: 24). Como puede observarse

\footnotetext{
${ }^{7}$ Los ejemplos que se indican a continuación están sacados de la versión de 1997 del relato en inglés publicada por Proulx en The New Yorker y de la primera traducción a castellano en España publicada por Siglo XXI de España en el año 2000, ambas reseñadas de forma completa en la sección de referencias.
} 
pues, en las alusiones principales a sexualidad en el texto la traductora opta por mantenerse fiel a lo expresado por Proulx, ni atenuando ni intensificando los encuentros ni las situaciones, no dejándose llevar por la intensidad de los mismos y adoptando una estrategia aséptica.

No obstante, no ocurrirá lo mismo con algunas de las expresiones malsonantes empleadas en el relato y que tanto sirven para caracterizar a los personajes: a continuación (véase tabla 1) observamos algunos de los casos más representativos en los que la traductora atenúa (casi hasta la supresión en algunos casos) las palabras de uno de los protagonistas, rebajando así el carácter de ofensa de sus expresiones. Se trata en todos los ejemplos del personaje de Jack, algo significativo que hace que siga siendo el menos masculino y "héroe" del relato, contribuyendo Corniero de este modo a aumentar la subversión que el relato de Proulx implica para el género del western.

\begin{tabular}{|l|l|}
\hline TÉCNICA: Atenuación & \\
\hline Texto original (1997) & Texto meta 1 (2000) \\
\hline $\begin{array}{l}\text { "Jesus Christ, quit hammerin and get } \\
\text { over here" (p.75) }\end{array}$ & "Dios mío, deja de dar la matraca \\
y vente aquí" (p. 9)
\end{tabular}

Tabla 1. Ejemplos de atenuación de expresiones malsonantes

Los ejemplos que pueden observarse en la tabla 1 muestran extractos de conversaciones entre Jack y Ennis, de modo que, atenuando las expresiones malsonantes utilizadas por el primero se enfatiza el carácter de macho dominante de Ennis, que es quien siempre parece ser el más rudo de los dos. Al mismo tiempo, resulta significativo que la traductora intensifique expresiones similares e igual de malsonantes cuando se trata de otros personajes masculinos presentes en el relato. Así ocurre, 
por ejemplo, con alguna expresión del padre de Jack cuando, una vez fallecido, Ennis acude a visitarle a él y a su madre:

\begin{tabular}{|l|l|}
\hline TÉCNICA: Intensificación & \\
\hline Texto original (1997) & Texto meta 1 (2000) \\
\hline $\begin{array}{l}\text { "He thought he was too goddamn } \\
\text { special to be buried in the family } \\
\text { plot" (p. } 84 \text { ) }\end{array}$ & $\begin{array}{l}\text { "El muy jodido se creía demasiado } \\
\text { la tumbal para que lo enterrásemos en }\end{array}$ \\
\hline
\end{tabular}

Tabla 2. Ejemplo de intensificación de expresiones malsonantes

Dicha estrategia por parte de la traductora refleja, e intensifica, la influencia del patriarcado que siempre había dominado y censurado la vida de ambos, en especial la de Jack, hasta cuando ya no está vivo: “el padre retiene a Jack en el cementerio de la familia como la sociedad lo retuvo en la familia durante toda su vida" (Averbach, 2009: 148).

La tendencia de Corniero a censurar las expresiones ofensivas de Jack y atenuarlas parece responder a esa predisposición frecuente de emplear una "manipulación inherentemente conservadora" ya mostrada en épocas anteriores (Franco Aixela \& Abio Villarig, 2009: 2) y que en este caso encaja como parte de la subversión del relato original. Esta traducción llevada a cabo por Corniero será la que sigue vigente en nuestro país una vez que la película ha llegado a las pantallas y ha alcanzado fama mundial. Tratándose de la misma traductora, lo esperable sería un mantenimiento de las estrategias de la primera edición. Sin embargo, un estudio detallado de las siguientes ediciones nos lleva a determinar que dicha traducción ha sido revisada: así, algunas de las expresiones que previamente había atenuado han pasado a adecuarse a la crudeza del texto inglés, haciendo caso omiso al hecho de que sean pronunciadas por Jack o no: en la tabla 3 se ofrecen los ejemplos de las mismas, en la edición de la obra publicada por Siglo XXI en 2005:

\begin{tabular}{|l|l|}
\hline TÉCNICA: transferencia & \\
\hline Texto original (1997) & Texto meta 2 (2005) \\
\hline $\begin{array}{l}\text { "Jesus Christ, quit hammerin and get } \\
\text { over here" (p. 75) }\end{array}$ & $\begin{array}{l}\text { "Coño, para ya esa matraca y vente } \\
\text { aquí" (p. 9) }\end{array}$ \\
\hline "I fucking knew it" (p. 78) & "Nos ha jodido que lo sabía" (p. 16) \\
\hline
\end{tabular}




\begin{tabular}{|c|c|}
\hline TÉCNICA: transferencia $^{8}$ & \\
\hline Texto original (1997) & Texto meta 2 (2005) \\
\hline "Fuckin starved" (p. 78) & "Pasé un hambre de cojones" (p. 17) \\
\hline $\begin{array}{l}\text { "We got us a fuckin situation here" } \\
\text { (p. 79) }\end{array}$ & $\begin{array}{l}\text { "Estamos metidos en un lío de } \\
\text { cojones" (p. 18) }\end{array}$ \\
\hline $\begin{array}{l}\text { "Where's the fuckin problem?" (p. } \\
83 \text { ) }\end{array}$ & “¿Algún problema, joder?” (p. 29) \\
\hline
\end{tabular}

Tabla 3. Ejemplo de transferencias de expresiones previamente atenuadas ${ }^{8}$

Como puede observarse en los ejemplos de la tabla 3, los expletivos que Jack Twist usa en inglés y que resultan tan malsonantes encuentran ahora su equivalencia en el texto transferido a castellano por Corniero. Estos se mantendrán también en la edición de la obra que Lumen hará en el 2009, donde se indica que la traducción ha sido cedida por Siglo XXI. El éxito que la película obtuvo en las pantallas fue seguramente lo que llevó a la traductora a revisar la primera versión en castellano que había realizado del relato, a sabiendas de que la tirada con la que iba a contar ahora la obra iba a ser mucho mayor. Una vez que la obra ha triunfado en el cine, donde ambos personajes son malsonantes (incluso mucho más en el doblaje que en la versión original, como se ha podido comprobar en el estudio de la traducción audiovisual -Pérez L. Heredia \& Gómez Castro. En prensa- y con un tipo de comunicación oral que define la rotundidad de sus actos (González Sánchez, 2011: 10), la traducción del relato puede pasar a reflejar la vulgaridad y los coloquialismos de los que Proulx dotó tanto a Jack como a Ennis sin necesidad de atenuar la crudeza de algunas de sus intervenciones. Si tal y como manifestaría Ennis en la película Brokeback Hit us Hard, puede afirmarse que los golpes que ellos recibieron y los que el relato atestó a la sociedad homofóbica del Oeste norteamericano han sido trasvasados a español de forma que mantienen su esencia, propósito final de toda actividad traductora.

\footnotetext{
${ }^{8}$ Por transferencia indicamos aquí una técnica de traducción consistente en transferir el discurso original a la lengua meta de forma que no se violen las normas de ninguno de los sistemas y el resultado sea un discurso equivalente: de esta manera los resultados de la transferencia apenas sí se distinguen de las producciones normales en la lengua meta (Toury, 1995: 275).
} 


\section{La película}

El trasvase intersemiótico, del relato al cine, también había sumido a Proulx en un mar de dudas y preocupaciones. Como sabemos, el relato contenía una serie de descripciones sexuales mucho más explícitas de lo que pudiera admitir la conservadora industria cinematográfica estadounidense. Había además otras cuestiones de importancia que dificultaban la reescritura a las pantallas: la homofobia, protagonista del relato y tan presente en la cultura y sociedad estadounidenses, el posible rechazo de los actores a interpretar a personajes gay, ante el evidente riesgo de estereotipación, sin olvidar el más que probable rechazo del público. El entusiasmo de Larry McMurtry y Diana Ossana al leer el relato, el mismo día en que The New Yorker lo publicó, contagió a la autora, que accedió, a pesar de todos los miedos, a que reescriberan el texto que terminaría ganando el Óscar al mejor guion adaptado del año 2005. Proulx quedó gratamente sorprendida del resultado, al constatar, en la nueva trama cinematográfica, la imaginación desplegada por los adaptadores, quienes añadieron algunos detalles con la finalidad de conservar (¿e intensificar?) el mensaje del texto original en el medio audiovisual. Solo quedaba encontrar productores que se arriesgaran económica e ideológicamente y, por supuesto, un realizador que respetara la esencia de relato y guion. Después de varios intentos infructuosos, el realizador taiwanés Ang Lee se atrevió a coger el timón y conducir el guion hasta el mejor puerto que cupiera esperar. Por si fuera poco, y contra todo pronóstico, el elenco protagonista era excepcional (capitaneados por Jack Gyllenhaal y el malogrado Heath Ledger en los papeles de Jack Twist y Ennis del Mar respectivamente) y la maestría de unos y otros convirtieron el texto fílmico en pura estética. Es sobresaliente, en términos de reescritura (y censura), el cambio que experimenta el clima de la historia: la sordidez que impregna el relato se torna en belleza. Todo en el texto fílmico aparece ante el espectador como bello: la fotografía, la música, el escenario albergan a los dos protagonistas que, como no puede ser de otra forma, son bellos, pulcros, a pesar de las vicisitudes y del entorno. El contraste entre relato y película, en cuanto a la descripción de los personajes, es más que evidente: 


\begin{tabular}{|l|l|}
\hline Proulx, 1997 & Lee, 2005 \\
\hline At first glance Jack seemed fair enough \\
with his curly hair and quick laugh, but \\
for a small man he carried some weight \\
in the haunch and his smile disclosed \\
buckteeth, not pronounced enough to let \\
him eat popcorn out of the neck of a jug, \\
but noticeable (p. 1). Jack Twist (Jake Gyllenhaal) \\
$\begin{array}{l}\text { Ennis, high-arched nose and narrow } \\
\text { face, was scruffy and a little cavechested, } \\
\text { balanced a small torso on long, caliper } \\
\text { legs, possessed a muscular and supple } \\
\text { body made for the horse and for fighting } \\
\text { (p. 1). }\end{array}$ \\
\hline
\end{tabular}

Tabla 4. Descripcion de los personajes

Sirva el ejemplo para demostrar que el proceso de estetización es más que significativo: ¿se trata de un caso de (auto)censura, más o menos consciente, por parte de los realizadores de la cinta? Desde un punto de vista de género, no parece que el eterno masculino, en el subconsciente de las y los espectadores, pudiera asimilar que los protagonistas resultaran sórdidos y llenos de defectos. En la misma línea, vamos a examinar dos ejemplos más, correspondientes al primer encuentro sexual entre los protagonistas y a su reencuentro, cuatro años después:

\section{Proulx, 1997}

In a little while they deepened their intimacy considerably. Ennis ran fullthrottle on all roads whether fence mending or money spending, and he wanted none of it when Jack seized his left hand and brought it to his erect cock. Ennis jerked his hand away as though he'd touched fire, got to his knees, unbuckled his belt, shoved his pants down, hauled Jack onto all fours and, with the help of the clear slick and a little spit, entered him, nothing he'd done before but no instruction manual needed. They went at it in silence except for a few sharp intakes of breath and Jack's choked "gun's goin off", then out, down, and asleep (p. 2-3). 


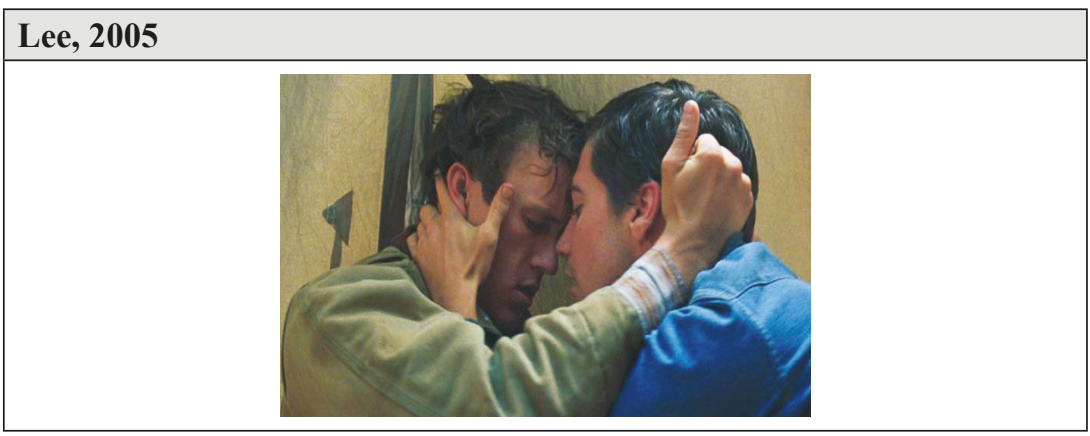

Tabla 5. Primer encuentro sexual de los personajes

\section{Proulx, 1997}

They seized each other by the shoulders, hugged mightily, squeezing the breath out of each other, saying, son of a bitch, son of a bitch, then, and easily as the right key turns the lock tumblers, their mouths came together, and hard, Jack's big teeth bringing blood, his hat falling to the floor, stubble rasping, wet saliva welling [...] Within twenty minutes were in the Motel Siesta jouncing a bed. A few handfuls of hail rattled against the window followed by rain and slippery wind banging the unsecured door of the next room then and through the night. The room stank of semen and smoke and sweat and whiskey, of old carpet and sour hay, saddle leather, shit and cheap soap. Ennis lay spread-eagled, spent and wet, breathing deep, still half tumescent (p. 6-7).

\section{Lee, 2005}

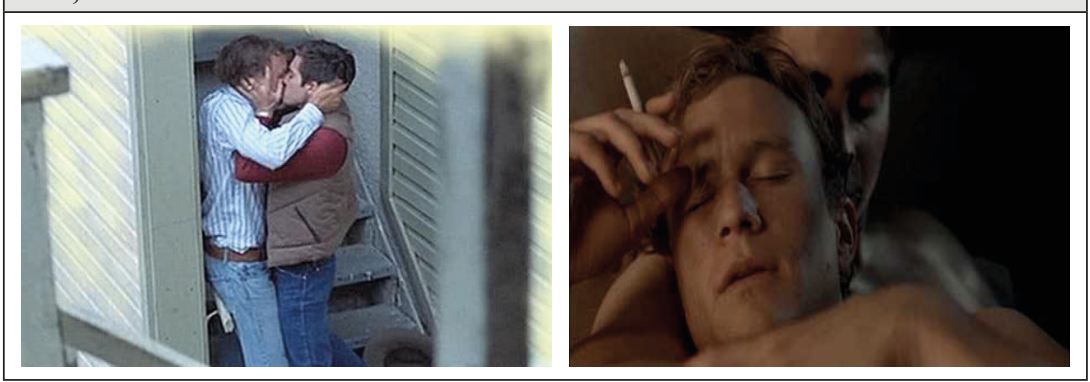

Tabla 6. Reencuentro de los personajes

Leemos una y otra vez las palabras de Proulx y, sin poder remediarlo, la sordidez de la descripción se apodera de nosotros cada vez más, y la historia nos atrapa. Las imágenes que reescriben las palabras, consi- 
guen el mismo efecto mostrando la representación icónica inversa. Se ahonda en esa idea de censura que, a través de la sublimación, más allá de la exploración de la belleza, busca la corrección política y la aprobación del público global que, cabe intuir, asiste atónito a la caída de los aguerridos arquetipos masculinos que habían protagonizado hasta entonces la historia del cine.

\section{Intersecciones (a modo de conclusión)}

Amada por unos y denostada por otros, la historia que Proulx tejió a finales de los noventa y que luego Lee trasvasó a la gran pantalla unos años después ha pasado a formar parte ya de la tradición del western en su desconstrucción o subversión del mismo y de la propia sociedad norteamericana. Tal y como ha podido verse a través de este estudio, tanto la historia como el relato han contado con versiones en otras sociedades y culturas gracias a la traducción o el doblaje y subtitulado. La actividad de trasvase cultural y lingüístico se ve afectada en su ejercicio por diversos factores que la condicionan, y que finalmente compone un todo que conduce al producto final. En este caso, los Jack Twist y Ennis del Mar que los lectores y espectadores españoles tuvieron (y tienen) la oportunidad de conocer difieren en algunos aspectos de los originales en inglés, bien por ser más moderados en sus expresiones o precisamente por lo contrario. Sea como fuere, esa misma fuerza que la naturaleza les imprime a ellos ha sido la que ha marcado a quienes los han conocido a través de la traducción, demostrandose así que esta ha cumplido su función.

$\mathrm{Y}$ es que, a pesar de no ser el resultado de una ingenua transferencia, la traducción de la historia de amor y sexo de los dos vaqueros supuso un impensable atrevimiento ideológico solo diez años atrás. Ello hizo que se tambalearan muchos viejos estereotipos y permitió convertir en norma lo que hasta entonces había sido provocación y subversion. Brokeback Mountain, definitivamente, abrió una ventana en el pensamiento y formato ideológico del nuevo tiempo audiovisual. Queda por ver si la naturalidad con la que se abordan, exhiben y reescriben hoy en día las cuestiones de género, presentadas en tantas ocasiones sublimando cánones impuestos de belleza y perfección, no terminan por convertirse en nuevos estereotipos que perpetúen el eterno (aunque tal vez desconocido) masculino. 


\section{Bibliografía}

Averbach, Márgara. 2009. Brokeback Mountain, el cuento y la película: la familia como infierno. Espacios de crítica y producción 42: 146-152.

Campbell, Neil. 2009. From story to film: Brokeback Mountain's "in-between" spaces. Canadian Review of American Studies 39(2): 205-220.

Cox, Christopher. 2009. Interview to Anne Proulx. The Paris Review, The Art of Fiction 199: http://www.theparisreview.org/interviews/5901/the-artof-fiction-no-199-annie-proulx [Acceso 20/11/2014].

Franco Aixelá, Javier \& Abio Villarig, Carlos. 2009. Manipulación ideológica y traducción: atenuación e intensificación moral en la traducción de la novela negra norteamericana al español (1933-2001). Hermeneus. Revista de Traducción e Interpretación 11: 1-23.

Gentzler, Edwin \& Tymoczko, Maria. 2002. Translation and Power. Amherst: University of Massachusetts Press.

González Sánchez, José Félix. 2011. Héroes, antihéroes y villanos en el western español. Razón y Palabra 78: 1-15.

Karger, Dave. 2006. Oscar's big night. Entertainment Weekly: http://www. ew.com/article/2006/03/10/oscars-big-night [Acceso 12/12/2014].

Lee, Ang. 2005. Brokeback Mountain. EE.UU.: Universal (guion de L. McMurtry y D. Ossana).

McDonald, Janet. A. 2007. Queering the representation of the masculine "west" in Ang Lee's Brokeback Mountain. Gay \& Lesbian Issues and Psychology Review 3(2): 79-85.

Proulx, Annie. 1997 (13 de octubre). Brokeback Mountain. The New Yorker, 74-85.

Proulx, Annie. 2000. En terreno vedado. Historias de Wyoming. Madrid: Siglo XXI de España. [Traducción de María Corniero].

Proulx, Annie. 2005. Brokeback Mountain. En terreno vedado. Historias de Wyoming. Madrid: Siglo XXI de España. [Traducción de María Corniero].

Proulx, Annie. 2009. Wyoming. Barcelona: Lumen. [Traducción de Brokeback Mountain de María Corniero, cedida por Siglo XXI de España].

Proulx, Annie; McMurtry, Larry \& Ossana, Diana. 2005. Brokeback Mountain: Story to Screenplay. Nueva York: Scribner (edición Kindle).

Roughton, Ralph. 2014. The significance of Brokeback Mountain. Journal of Gay \& Lesbian Mental Health 18(1): 83-94.

Shugart, Helene A. 2011. Consuming passions: "Educating desire" in Brokeback Mountain. Critical Studies in Media Communication 28(3): 173192. 
Spohrer, Erika. 2009. Not a gay cowboy movie? Journal of Popular Film and Television 37(1): 26-33.

Toury, Gideon. 1995. Descriptive Translation Studies and Beyond. Ámsterdam: John Benjamins.

Verdú, Vicente. 1996. El planeta americano. Barcelona: Anagrama. 